\title{
STAT3 Genotypic Variant rs744166 and Increased Tyrosine Phosphorylation of STAT3 in IL-23 Responsive Innate Lymphoid Cells during Pathogenesis of Crohn's Disease
}

\author{
Ying Tang $\mathbb{D},{ }^{1}$ Sanda A. Tan $\left(\mathbb{D},{ }^{2}\right.$ Atif Iqbal, ${ }^{3}$ Jian Li, ${ }^{4}$ and Sarah C. Glover $\mathbb{D}^{1}$ \\ ${ }^{1}$ Department of Medicine, University of Florida, Gainesville, FL 32610, USA \\ ${ }^{2}$ Department of Surgery, University of Florida, Gainesville, FL 32610, USA \\ ${ }^{3}$ Department of Surgery, Baylor College of Medicine, Houston, TX 77030, USA \\ ${ }^{4}$ Department of Neurology, Thomas Jefferson University, Philadelphia, PA 19107, USA
}

Correspondence should be addressed to Sarah C. Glover; sarah.glover@medicine.ufl.edu

Received 22 March 2019; Revised 17 May 2019; Accepted 27 May 2019; Published 19 June 2019

Guest Editor: Erwei Sun

Copyright (c) 2019 Ying Tang et al. This is an open access article distributed under the Creative Commons Attribution License, which permits unrestricted use, distribution, and reproduction in any medium, provided the original work is properly cited.

Crohn's disease (CD) results from dysregulated immune responses to gut microbiota in genetically susceptible individuals, affecting multiple areas of the gastrointestinal tract. Innate lymphoid cells (ILCs) are tissue-resident innate effector lymphocytes which play crucial roles in mucosal immune defense, tissue repair, and maintenance of homeostasis. The accumulation of IFN- $\gamma$-producing ILC1s and increased level of proinflammatory cytokines produced by ILCs has been observed in the inflamed terminal ileum of CD patients. To date, the precise mechanisms of ILC plasticity and gene regulatory pathways in ILCs remain unclear. Signal transducer and activator of transcription 3 (STAT3) regulates gene expression in a cell-specific, cytokine-dependent manner, involving multiple immune responses. This study proposes the positive correlation between the prevalence of STAT3 rs744166 risky allele "A" with the severity of disease in a cohort of $94 \mathrm{CD}$ patients. In addition, the results suggest an increased STAT3 activity in the inflamed ileum of CD patients, compared to unaffected ileum sections. Notably, IL-23 triggers the differentiation of $\mathrm{CD} 117^{+} \mathrm{NKp} 44^{-}$ILC3s and induces the activation of STAT3 in both CD117 $7^{+} \mathrm{Np} 44^{-}$and CD117 NKp44- ILC subsets, implying the involvement of STAT3 in the initiation of ILC plasticity. Moreover, carriage of STAT3 "A" risk allele exhibited a higher basal level of STAT3 tyrosine phosphorylation, and an increased IL-23 triggered the pSTAT3 level. We also demonstrated that there was no delayed dephosphorylation of STAT3 in ILCs of both A/A and G/G donors. Overall, the results of this study suggest that IL-23-induced activation of STAT3 in the CD117-NKp44- ILC1s involves in ILC1-toILC3 plasticity and a potential regulatory role of ILC1 function. Those genetically susceptible individuals carried STAT3 rs744166 risky allele appear to have higher basal and cytokine-stimulated activation of STAT3 signal, leading to prolonged inflammation and chronic relapse.

\section{Introduction}

Crohn's disease (CD) is one type of inflammatory bowel disease (IBD) that involves a chronic relapsing inflammation of the gastrointestinal tract, causing abdominal pain and diarrhea and can eventually lead to severe strictures or fistulae which enhance the need for surgical intervention [1]. The pathogenesis of $\mathrm{CD}$ involves a complex interaction between genetic factors, environmental factors, and dysregulated immune responses [2]. To date, the precise mechanisms behind the disease initiation and progression remain unclear. However, it is widely accepted that CD involves an inappropriate and continuing mucosal inflammatory immune response to intestinal microflora in genetically susceptible individuals $[3,4]$. Historically, the dysfunctional adaptive immune responses were investigated in terms of Th1/Th17 responses in $\mathrm{CD}$ patients, and results suggested that $\mathrm{T}$ cells are responsible for the increased production of proinflammatory cytokines, such as IFN- $\gamma$ and IL-17A [5]. Recently, increasing evidence suggests that innate immunity also plays 
a significant role in the pathogenesis of $\mathrm{CD}[6,7]$. Innate lymphoid cells (ILCs) have been identified as innate effector cells that are involved in bacterial defense, induction of inflammation, and the maintenance of homeostasis [8]. The nomenclature of ILCs is based on their signature cytokines and the transcriptional factors that regulate their differentiation. They are classified into natural killer (NK) cells, group 1 ILCs (ILC1s), group 2 ILCs (ILC2s), group 3 ILCs (ILC3s), and lymphoid tissue inducer (LTi) cells [9]. In contrast to T cells, ILCs lack antigen-specific receptors and are constitutively located in barrier tissues, responding to alarmins as well as cytokine signals released following tissue damage or mucosal infection $[10,11]$. As tissue-resident innate compartments of T helper cells, ILCs undergo transdifferentiation, also known as plasticity, in response to cytokine cues in the surrounding tissue microenvironment [12]. Specifically, in Crohn's disease patients, we and other researchers have identified the accumulation of IFN- $\gamma$-producing ILC1s at the expense of IL-17/IL-22-producing ILC3s in the inflamed intestinal tissues of Crohn's disease patients, implying this ILC3-toILC1 plasticity may be involved in the CD pathogenesis $[13,14]$. Moreover, researchers have identified that the differentiation toward ILC1s was driven by IL-12, and conversely, IL-23 promoted polarization toward ILC3s in vitro and in vivo [15]. Notably, IL-23 responsive ILCs have been recently recognized as the important cell population that associates with the pathogenesis of colitis in several murine models [16].

Genome-wide association studies (GWAS) have identified 170 disease susceptibility gene loci that are associated with Crohn's disease [17]. In 2014, a meta-analysis indicated that the STAT3 rs744166 polymorphism was highly associated with $C D$ susceptibility, especially among Caucasians [18]. Additionally, the STAT3 rs744166 risk allele "A" has been associated with increased cellular STAT3 activation in leukocytes in pediatric Crohn's disease patients [19]. Signal transducer and activator of transcription 3 (STAT3) is a crucial transcription factor which regulates cells associated with both innate and adaptive mucosal immunity in a cell-specific, cytokine-dependent manner [20]. Particularly, STAT3 plays a central role in Th17 commitment through its direct regulation of ROR $\gamma \mathrm{t}$ and regulates Th17-cytokine production in response to IL-23 [21]. In addition, it has been shown that STAT3 is essential for ILC3s to produce IL-22 against intestinal infection in mice $[22,23]$. Recent improvements in genetic analysis in human studies have revealed the distinct gene regulatory mechanisms in adaptive and innate lymphoid cells $[24,25]$. Although ILC3s are the innate analog of Th17 cells, the exact regulation of STAT3 in ILC3 development and plasticity remains unclear. In this study, we investigate the role of STAT3 in the pathogenesis and progression of Crohn's disease, and more specifically, we examine the STAT3 signaling involving in innate lymphoid cell plasticity.

\section{Materials and Methods}

2.1. Human Intestinal Lamina Propria Mononuclear Cell (LPMC) Isolation. All the surgical resections of the terminal ileum from Crohn's disease patients were obtained from
TABLE 1: STAT3 rs744166 genotyping in a cohort of Crohn's disease patients.

\begin{tabular}{lcc}
\hline Genotype & Number & Frequency \\
\hline GG & 12 & $12.8 \%$ \\
GA & 41 & $43.6 \%$ \\
AA & 41 & $43.6 \%$ \\
\hline
\end{tabular}

UF Health Shands Hospital, following an approved IRB\#201500440. LPMCs were isolated by epithelial elimination and enzyme digestion. Briefly, the surgical resections were cut into 1-2 $\mathrm{mm}$ pieces after removal of the outer fat tissue and muscle layer. The epithelial layer was removed by $1 \mathrm{X}$ Hanks' Balanced Salt Solution (HBSS) with phenol red and glucose, no calcium, and no magnesium, containing $2 \%$ FBS $\left(\mathrm{Gibco}^{\circledR}\right), 100 \mathrm{U} / \mathrm{ml}$ penicillin, $100 \mu \mathrm{g} / \mathrm{ml}$ streptomycin, $25 \mu \mathrm{g} / \mathrm{ml}$ gentamycin, and 0.5 $\mathrm{mM}$ DTT. Tissues were then digested with $1 \mathrm{X}$ HBSS containing $1 \%$ FBS $\left(\mathrm{Gibco}^{\circledR}\right), 100 \mathrm{U} / \mathrm{ml}$ collagenase XI (Roche), $20 \mu \mathrm{g} / \mathrm{ml}$ Dispase neutral protease II (Roche), and $10 \mu \mathrm{g} / \mathrm{ml}$ deoxyribonuclease I (StemCell Technologies). The remaining tissues were mechanically dissociated and were passed through $70 \mu \mathrm{m}$ cell strainers (Corning ${ }^{\circledR}$ ). All cells were then slowly cryopreserved in CryoStor ${ }^{\circledR}$ CS10 (StemCell Technologies) and transferred into liquid nitrogen for future use.

\subsection{Human Peripheral Blood Mononuclear Cell (PBMC)} Isolation. Donor blood was purchased from LifeSouth Community Blood Center, and patient blood was obtained from UF Health Shands Hospital, following an approved IRB\#201500440. PBMCs were isolated by gradient centrifugation. Briefly, whole blood samples were diluted 1:1 ratio with 1 XPBS containing $2 \%$ FBS. Diluted blood samples were carefully transferred into SepMate ${ }^{\mathrm{TM}}-50$ tubes containing 15 $\mathrm{ml}$ of Lymphoprep ${ }^{\mathrm{TM}}$ (StemCell Technologies). Samples were then centrifuged at $1,200 \mathrm{xg}$ for $10 \mathrm{mins}$, and the cell suspensions above the insert in SepMate ${ }^{\mathrm{TM}}-50$ tubes were transferred into new $50 \mathrm{ml}$ conical tubes. PBMCs were collected by centrifuging at $300 \mathrm{xg}$ for 8 mins. All cells were then slowly cryopreserved in CryoStor ${ }^{\circledR}$ CS10 (StemCell Technologies) and transferred into liquid nitrogen for future use.

2.3. Genotyping of STAT3 rs744166. Genomic DNA was extracted from whole blood using the Gentra Puregene Kit (QIAGEN). Patients were genotyped for the STAT3 rs744166 (assay ID: C__3140282_10, Cat. No. 4351379, TaqMan) single nucleotide polymorphism (SNP) using the TaqMan system, running on the QuantStudio 7 Flex RealTime PCR System (Applied Biosystems).

2.4. Quantitative RT-PCR. Total RNA was isolated from intestinal tissues of Crohn's disease patients using QIAzol Lysis Reagent (QIAGEN) or from whole blood of CD patients using the RNeasy Kit (QIAGEN). A two-step RTPCR was performed using the SuperScript ${ }^{\circledR}$ VILO cDNA Synthesis Kit (Thermo Fisher Scientific) for reverse transcription (RT), and PCR was done using the QuantStudio 7 
TABLE 2: Surgery numbers in risky allele carrier and nonrisky allele carrier among the cohort of Crohn's disease patients.

\begin{tabular}{lccccccccc}
\hline \multirow{2}{*}{ Genotype } & & & \multicolumn{3}{c}{ Surgery numbers } & & & \\
& & 0 & 1 & 2 & 3 & 4 & 5 & 0 \\
\hline \multirow{2}{*}{ GG } & Number & 9 & 3 & 0 & 0 & 0 & 0 & 12 \\
& Percentage & $9.57 \%$ & $3.19 \%$ & $0 \%$ & $0 \%$ & $0 \%$ & $0 \%$ & $0 \%$ & $12.77 \%$ \\
\hline \multirow{2}{*}{ AA+GA } & Number & 26 & 21 & 11 & 9 & 8 & 6 & 1 & 82 \\
& Percentage & $27.66 \%$ & $22.34 \%$ & $11.70 \%$ & $9.57 \%$ & $8.57 \%$ & $6.38 \%$ & $1.06 \%$ & $87.23 \%$ \\
\hline \multirow{2}{*}{ Total } & Number & 35 & 24 & 11 & 9 & 8 & 6 & 1 & 94 \\
& Percentage & $37.23 \%$ & $25.53 \%$ & $11.70 \%$ & $9.57 \%$ & $8.51 \%$ & $6.38 \%$ & $1.06 \%$ & $100 \%$ \\
\hline
\end{tabular}

Flex Real-Time PCR System (Applied Biosystems). The PCR was performed using a $20 \mu \mathrm{l}$ reaction volume containing the cDNA, $2 \times$ TaqMan ${ }^{\circledR}$ Gene Expression Master Mix, nucleasefree water, and $1 \times$ TaqMan Gene Expression Assay for STAT3 (Thermo Fisher Scientific). The housekeeping gene GAPDH was used as an endogenous control to normalize target gene expression levels. $2^{\wedge}-\Delta \Delta \mathrm{Ct}$ value was calculated and used to represent the gene expression fold change.

2.5. Immunohistochemistry. The immunohistochemistry staining followed the manufacturer's protocols, and the heat-induced epitope retrieval method with citrate buffer was used for antigen retrieval. Primary antibodies, specifically anti-STAT3 (Y705) antibody (rabbit polyclonal, ab214465 (dilution 1:500), Abcam), were added and incubated at $4^{\circ} \mathrm{C}$ overnight. The primary antibodies were detected using a mouse and rabbit specific HRP/DAB IHC detection kit (ab236466, Abcam) following the manufacturer's instructions.

2.6. Flow Cytometry. Single-cell suspensions isolated from intestinal tissues of $\mathrm{CD}$ patients were stained with FITCconjugated anti-human lineage cocktail 3 (Lin3) (CD3, CD14, CD19, and CD20; clone: M $\varphi$ P9, L27, SK7, and SJ25C1; BD Biosciences), PerCP-conjugated anti-human CD45 (clone: HI30, BioLegend), APC-conjugated antihuman CD127 (clone: A019D5, BioLegend), PE-conjugated anti-human NKp44 (clone: P44-8, BioLegend), PE-Cy7conjugated anti-human CD117 (clone: 104D2, eBioscience), Brilliant Violet 421-conjugated anti-human CRTH2 (clone: BM16, BioLegend), and Alexa Fluor 647-conjugated antiSTAT3 Phospho-Tyr705 (clone: 13A3-1, BioLegend). Viability was assessed by Fixable Yellow Dead Cell Stain (Thermo Fisher Scientific). For phosphoflow staining, cells were stimulated with $20 \mathrm{ng} / \mathrm{ml}$ of recombinant human IL-23 (carrier free, R\&D) for 15 mins and were permeabilized by TruePhos $^{\mathrm{TM}}$ Perm Buffer (BioLegend) following the manufacturer's protocol. AbC ${ }^{\mathrm{TM}}$ Total Antibody Compensation Beads (Cat. No. A10497, Life Technologies) and $\mathrm{ArC}^{\mathrm{TM}}$ Amine Reactive Compensation Beads (Cat. No. A10346, Life Technologies) were used for compensation. Data were acquired on an LSRFortessa ${ }^{\mathrm{TM}}$ flow cytometer (BD Biosciences) using BD FACSDiva ${ }^{\mathrm{TM}}$ software in the Cytometry Core at the University of Florida and analyzed by FlowJo software (Version 10, Tree Star Inc.).
2.7. Histology. The surgical tissues containing the structures of mucosa, submucosa, and muscular layer were fixed in $10 \%$ neutral-buffered formalin (Fisher Scientific) for at least $18 \mathrm{hrs}$. The fixed tissues were then embedded in paraffin, sectioned, and stained with hematoxylin and eosin.

2.8. Gene Expression Heatmap. Total RNA was isolated from intestinal tissues of Crohn's disease patients using QIAzol Lysis Reagent (QIAGEN), and a two-step RT-PCR was performed using the SuperScript ${ }^{\circledR}$ VILO cDNA Synthesis Kit (Thermo Fisher Scientific) for reverse transcription (RT). Quantitative RT-PCR was done using TaqMan ${ }^{\circledR}$ Gene Expression Array Plates (96-well Standard Human Immune Response Array, Cat. No. 4414073, TaqMan) on the QuantStudio 7 Flex Real-Time PCR System (Applied Biosystems). The expression levels of each gene were calculated using the $2^{\wedge}-\Delta \Delta \mathrm{Ct}$ value, and GAPDH was used as endogenous control gene whereas normal unaffected tissue sample was used as reference control. Heatmap of RT-PCR data was generated using $\mathrm{R}$ programming.

2.9. Statistical Analysis. The correlation of surgery number and STAT3 rs744166 risk allele was tested by a GENMOD procedure in SAS with the adjustment of age at diagnosis. Statistical analysis was performed using GraphPad Prism 6 software (GraphPad Software, CA), and all comparisons between experimental groups were evaluated by the unpaired Mann-Whitney $t$ test. Differences with a value of $p<0.05$ were considered as significant and were indicated by an asterisk.

\section{Results}

3.1. STAT3 rs744166 Risk Allele " $A$ " Carriage Positively Correlates with Clinical Outcomes of Crohn's Disease Patients. Surgery is not commonly required for CD patients; however, depending on disease severity and chronic relapse of inflammation, surgical removal of the inflamed tissues is needed in certain cases. Even though the carriage of STAT3 rs744166 risk "A" allele is relatively high in healthy individuals, which is $56.4 \%$ compared to $63.6 \%$ carriage in CD patients [26], it has been indicated in several studies that STAT3 rs744166 is associated with Crohn's disease susceptibility $[18,27]$. Herein, we first genotyped our cohort of $94 \mathrm{CD}$ patients and found that $87.2 \%$ of this cohort carried STAT3 rs744166 risk allele "A," heterozygous or homozygous (Table 1). In order to 

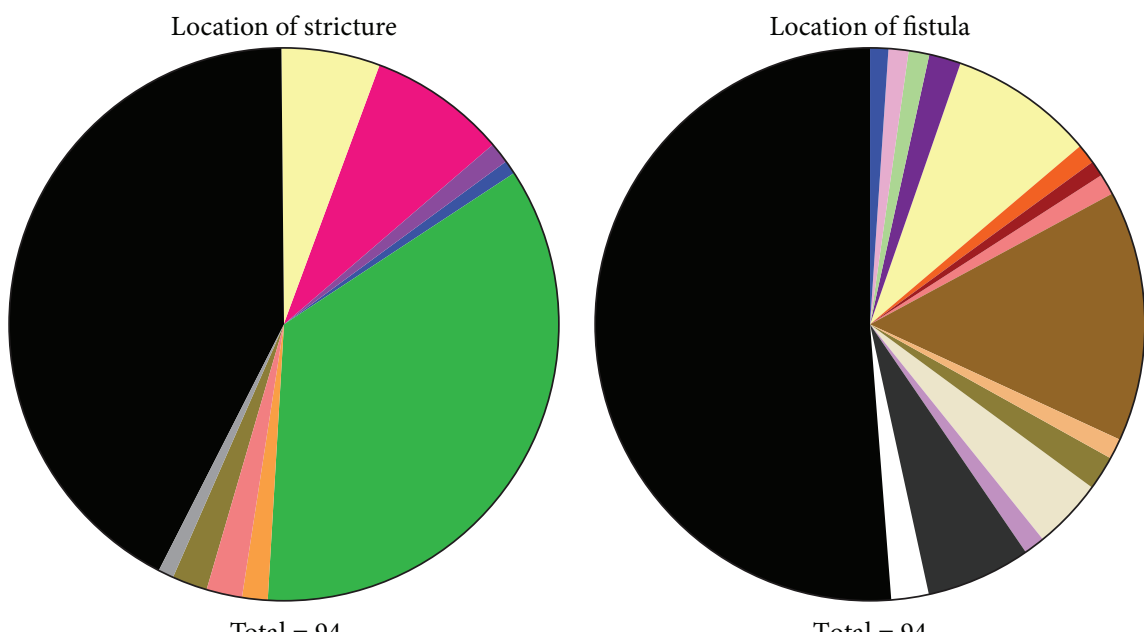

Total $=94$
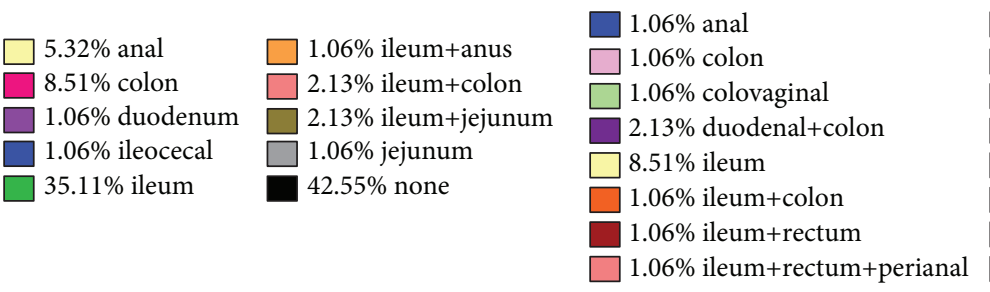

$14.89 \%$ perianal $1.06 \%$ perianal+ileum $2.13 \%$ perianal+ileum + colon $4.26 \%$ perianal+rectum $1.06 \%$ perirectal $6.38 \%$ rectum $2.13 \%$ rectum+perianal

(a)

(b)

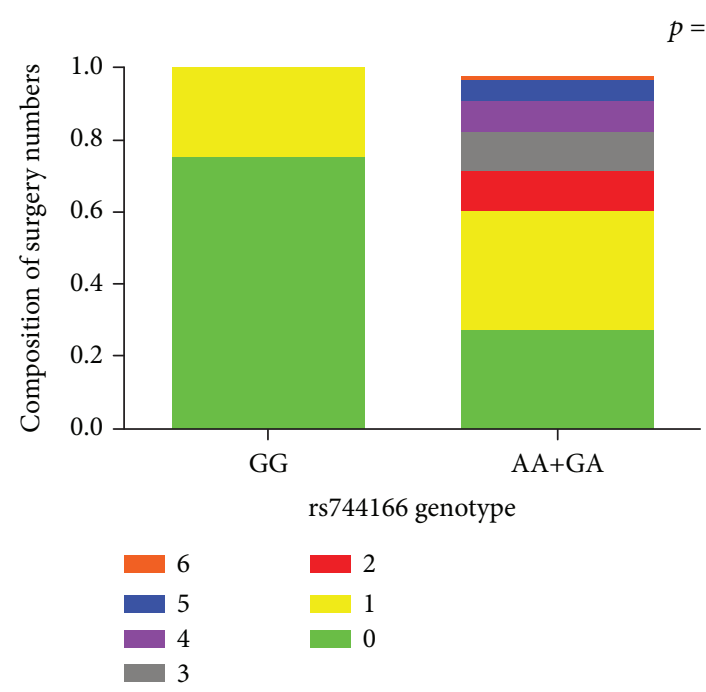

(c)

FIGURE 1: STAT3 rs744166 risky allele "A" positively correlates with surgery numbers in Crohn's disease patients. Patients in this study suffered stricture and fistula at various sites of the gastrointestinal tract. The locations of strictures was shown in (a), and (b) demonstrated various locations of fistula in this cohort of patients. (c) The correlation of surgery numbers and carriage of risky allele "A" was tested by a GENMOD procedure in SAS with the adjustment of age of diagnosis. Different surgery numbers were represented as different colors.

investigate whether this STAT3 single nucleotide polymorphism (SNP) was associated with clinical outcomes (Supplementary Table 1), we used the number of surgeries that these patients received after diagnosis to represent disease severity (Table 2). We observed that more than $60 \%$ of patients do not respond well to drug treatment and eventually need surgery. Moreover, $37.22 \%$ of patients have received multiple surgeries (surgery numbers $\geq 2$ ). In addition, $57.45 \%$ of patients have strictures located in various sites, and $48.94 \%$ of patients suffered from fistula at different locations (Figures 1(a) and 1(b)). However, there is no correlation between STAT3 rs744166 and the presence of stricture or fistula. Furthermore, a statistical correlation test of numbers of surgeries and the SNP was performed, and we found that there was a positive correlation between STAT3 rs744166 risky allele carriage (G/A or A/A) and surgery numbers (Figure 1(c)). Taken together, these results implied a potential involvement of dysfunctional STAT3 activity in the chronic relapse of Crohn's disease patients. 


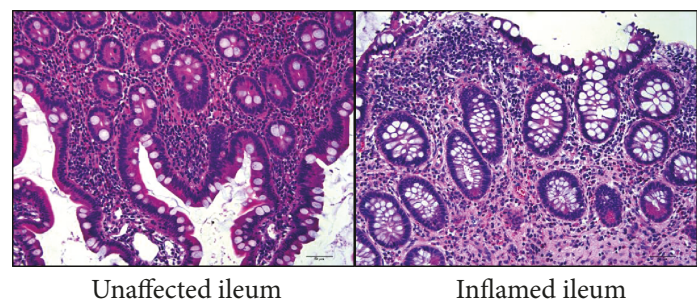

(a)

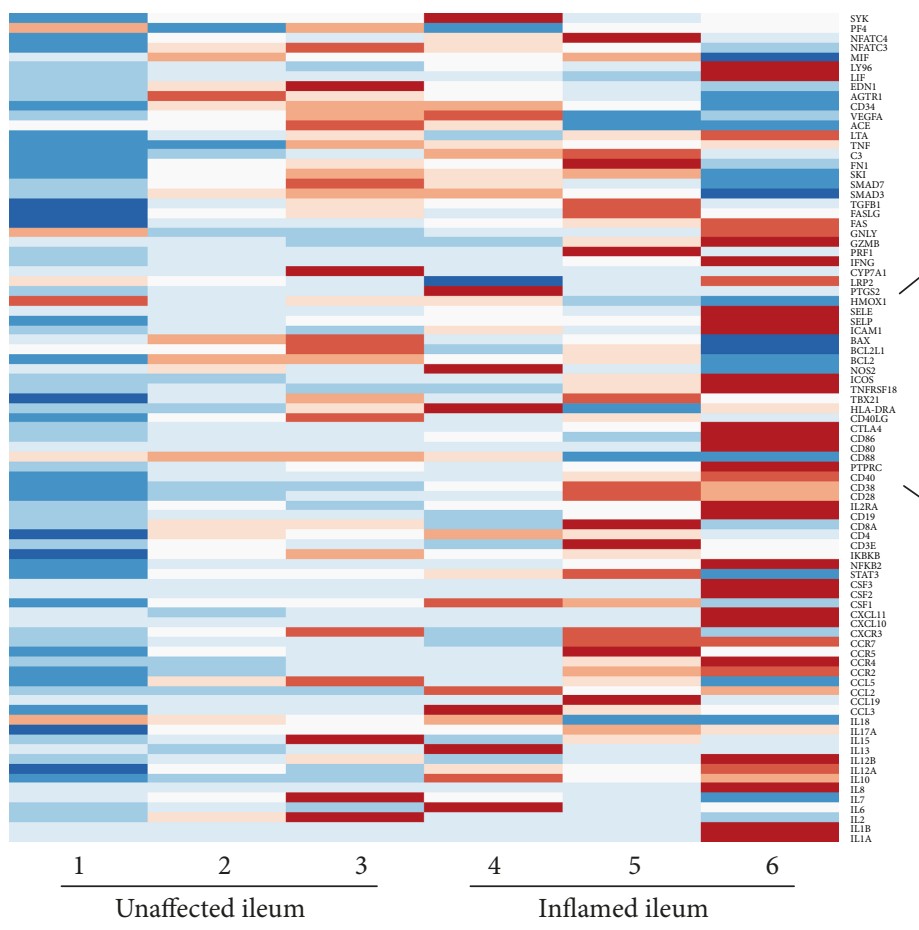

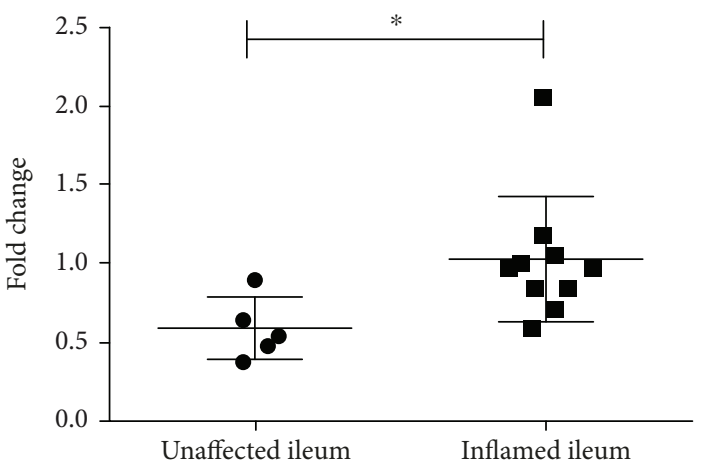

(b)

(c)

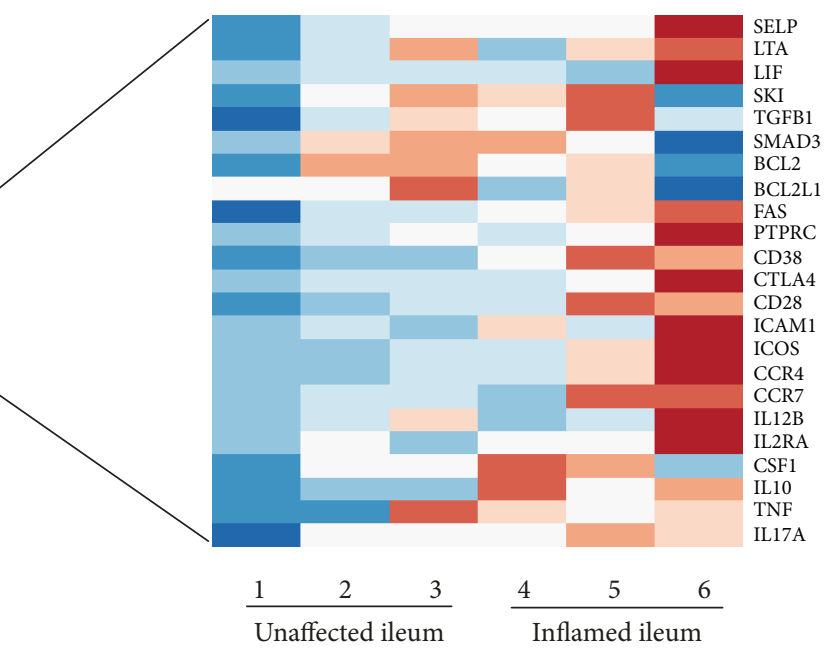

Color key

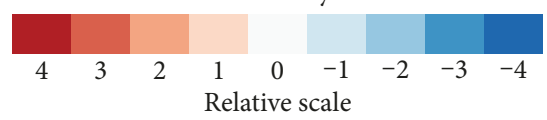

FIGURE 2: Increased STAT3 target gene expression and stat3 mRNA expression in the inflamed ileum of Crohn's disease patients. (a) Representative H\&E staining illustrates histological appearance in unaffected and inflamed terminal ileum of CD patients. Compared to unaffected ileum, the inflamed ileum showed distinct histological signs of inflammation, including increased infiltration of immune cells in the lamina propria, loss of intact small intestinal architecture, and ulceration. Images were obtained under 20x magnification. Scale bar $=50 \mu \mathrm{m}$. (b) stat 3 mRNA expression was upregulated in the inflamed ileum of CD patients $(n=10)$, compared to unaffected ileum tissues $(n=5)$. The gene expression fold change was calculated as $2^{\wedge}-\Delta \Delta \mathrm{Ct}$ value, using GAPDH as endogenous control. Statistical analysis was done by the unpaired Mann-Whitney $t$ test, $\alpha=0.05$. (c) Altered expression pattern of inflammatory genes in the inflamed tissues of CD patients. Among these genes, an increased STAT3-related gene expression was observed in the inflamed ileum of CD patients. GAPDH was used as endogenous control.

3.2. Increased stat 3 and Its Target Gene Expression in the Inflamed Ileum of Crohn's Disease Patients. To further investigate STAT3 activity in the disease state, we evaluated stat3 mRNA levels in the inflamed ileum tissues from CD patients who received more than 2 surgeries. Based on distinct histological features, for instance, ulceration, crypt abscesses, lymphoid aggregates, and architectural distortion, we defined surgical resections from CD patients as microscopic unaffected ileum and inflamed ileum (Figure 2(a)). We observed a significantly increased level of stat 3 mRNA expression in the inflamed ileums, compared to unaffected ileum of CD patients (Figure 2(b)), suggesting STAT3 activity was upregulated under inflammation. In addition, the stat 3 expression level was also increased in the peripheral blood of CD patients, compared to healthy donors (Supplementary Figure 1). Furthermore, an altered expression pattern of inflammatory genes was observed in the inflamed ileum of CD patients, compared to unaffected ileum (Figure 2(c), A). Among those inflammatory genes, we further evaluated the STAT3-related genes, such as Il-17a, tnf, and Il-10, and 


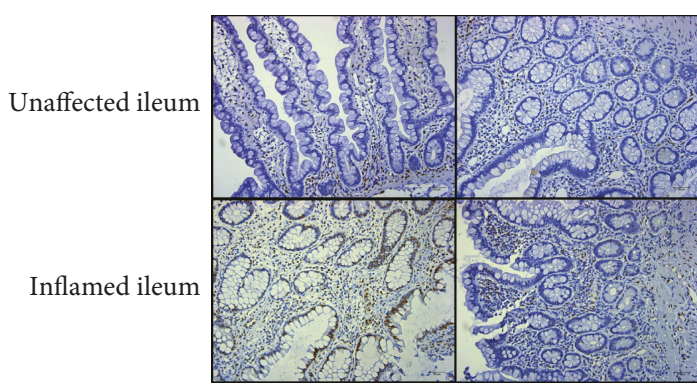

(a)

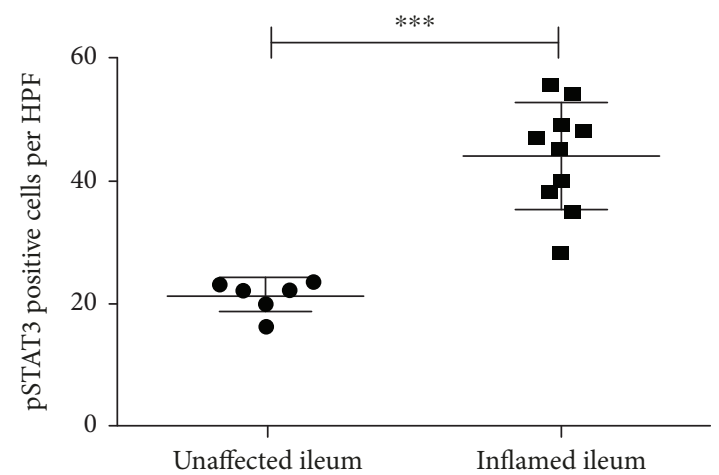

(b)

FIGURE 3: Increased activation of STAT3 signaling in the inflamed ileum of Crohn's disease patients. (a) Representative images of pSTAT3Y705 IHC staining on unaffected $(n=6)$ and inflamed ileum of CD patients $(n=10)$. pSTAT3 ${ }^{+}$cells were shown as brown dots. Images were obtained under 20x magnification. Scale bar $=50 \mu \mathrm{m}$. (b) Statistical analysis of pSTAT3-Y705 ${ }^{+}$cells per HPF in unaffected and inflamed ileum of CD patients. Unpaired Mann-Whitney $t$ test was performed, $\alpha=0.05$.

found increased expression levels of those STAT3 target genes in inflamed ileum tissues (Figure 2(c), B). The evaluated gene IDs were listed in Supplementary Table 2, and the calculated expression levels of each gene were reported in Supplementary Table 3. In order to investigate whether STAT3 rs744166 associates with the increase of STAT3-related gene expression during inflammation, we then genotyped these patients. As the result, the genotype of sample 1,2, and 5 was A/A, and the genotype of sample 6 was GG. Samples 3 and 4 were the same individual before and after treatment of 5-month Ustekinumab (anti-IL12/23 p40 antibody), and this patient was a heterozygous " $\mathrm{A}$ " carrier. However, the data implied this increased proinflammatory gene expression level was not associated with STAT3 rs744166 risk allele "A" but correlated with the inflammation level.

3.3. Increased Activation of STAT3 Signaling in the Inflamed Ileum of Crohn's Disease Patients. To examine the activation of STAT3 signaling, we performed IHC staining of pSTAT3Y705 on paraffin-embedded tissue sections of CD patients. The phosphorylated STAT3 signal was detected mainly in those immune cells located in the lamina propria, as well as intestinal epithelial cells for tissue repair in the inflamed ileum [28] (Figure 3(a)). pSTAT3 ${ }^{+}$cells were counted per high-power field (HPF) in unaffected tissues and inflamed tissues of CD patients, and an increased activation of STAT3 was observed in the inflamed ileum (Figure 3(b)).

3.4. Activation of STAT3 Signaling in Innate Lymphoid Cells. STAT3 has been shown that plays a critical role in Th17 development and function, responding to IL-6 and IL-23 signals. As the innate compartment of T helper cells, ILCs also respond to IL-23 stimulation, and the activation of STAT3 was crucial to the IL-22 production of ILC3s [22]. However, it is unknown whether STAT3 involves in ILC1-to-ILC3 transdifferentiation as IL-23 is the driving cytokine for this plasticity [15]. We have observed differential frequency of Lineage ${ }^{-} \mathrm{CRTH} 2^{-} \mathrm{CD} 45^{+} \mathrm{NKp} 44^{-} \mathrm{CD} 117^{-} \mathrm{CD} 127^{+}$ILC subset in the inflamed terminal ileum of CD patients [13], suggest- ing there was a dysregulated ILC plasticity in CD patients. Herein, we evaluated pSTAT3 levels in ILC subsets in response to IL-23 stimulation using PBMCs from healthy donor, and we also desired to investigate whether the carriage of STAT3 rs744166 "A" allele correlates with the altered activation level of STAT3 in ILCs. As the result, the NCR ${ }^{-}$ILC3s ( in $^{-} \mathrm{CD} 45^{+} \mathrm{CD}^{-} 6^{-} \mathrm{CD} 127^{+} \mathrm{CRTH} 2^{-} \mathrm{CD} 117^{+} \mathrm{NKp} 44^{-} \quad$ cells) responded rapidly to IL-23 stimulation (Figure 4(a)), and these cells are more progenitor-like cells which are able to differentiate to $\mathrm{NCR}^{+}$ILC3s [29]. We then examined the phosphorylation level of STAT3 following IL-23 in each ILC subset (Figure 4(b)). To our surprise, the pSTAT3 signal was also detected in the $\mathrm{Lin}^{-} \mathrm{CD} 45^{+} \mathrm{CD} 56^{+} \mathrm{CD} 127^{+-}$ $\mathrm{CRTH}^{-} \mathrm{CD} 117^{-} \mathrm{NKp} 44^{-}$ILC1s, which were reported accumulated in the inflamed ileum of CD patients [13]. This result suggested that the activation of STAT3 signaling may be the first step of a signaling cascade for ILC1-toILC3 plasticity and implied that STAT3 potentially has a regulatory role for ILC1 functioning. Furthermore, we eager to investigate whether the STAT3 genotypic variant has impact on cellular STAT3 activation in ILCs in response to IL-23. Here, we used PBMCs from donors with $\mathrm{A} / \mathrm{A}$ and $\mathrm{G} / \mathrm{G}$ genotypes to evaluate the potential effect of STAT3 "A" risk allele carriage on the phosphorylation of STAT3 in ILCs. Notably, the result revealed that donors carrying STAT3 rs744166 "A" risk allele exhibited a higher basal level of STAT3 tyrosine phosphorylation as well as an increased IL-23-stimulated pSTAT3 level in $\mathrm{Lin}^{-} \mathrm{CD} 45^{+} \mathrm{CD} 56^{+} \mathrm{CD} 127^{+} \mathrm{CRTH} 2^{-}$ILCs (Figure 4(c), A: A/A donor; B: G/G donor). By examination of pSTAT3 after 15 mins and 2 hrs of IL-23 stimulation, we illustrated that there was no delayed dephosphorylation of STAT3 in ILCs. In addition, the carriage of "A" allele did not impact the dephosphorylation of STAT3.

\section{Discussion}

Despite the introduction of numerous biological agents, including anti-TNFs, anti-integrin blockers, and more recently, anti-IL12/23 drugs, for Crohn's disease patients in 


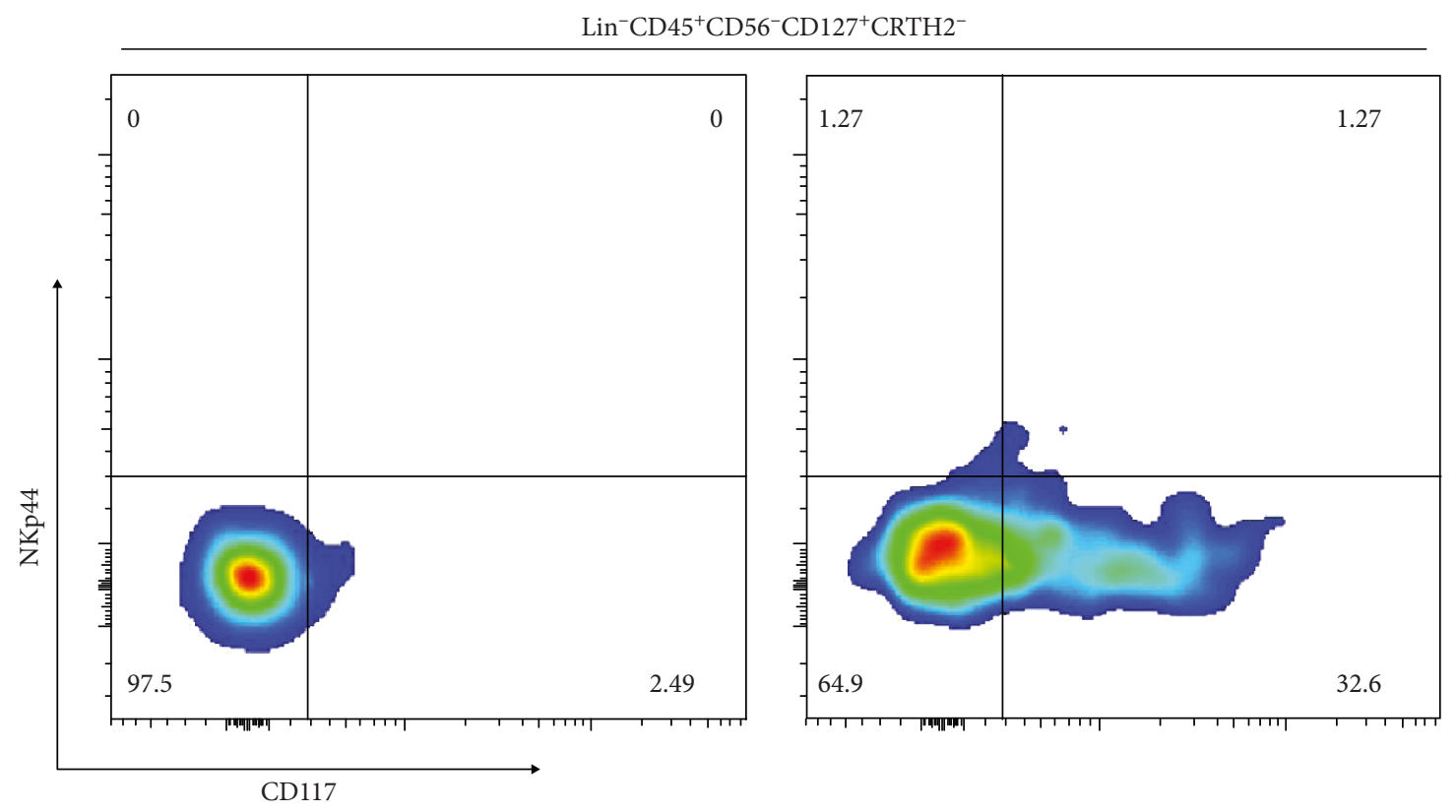

(a)

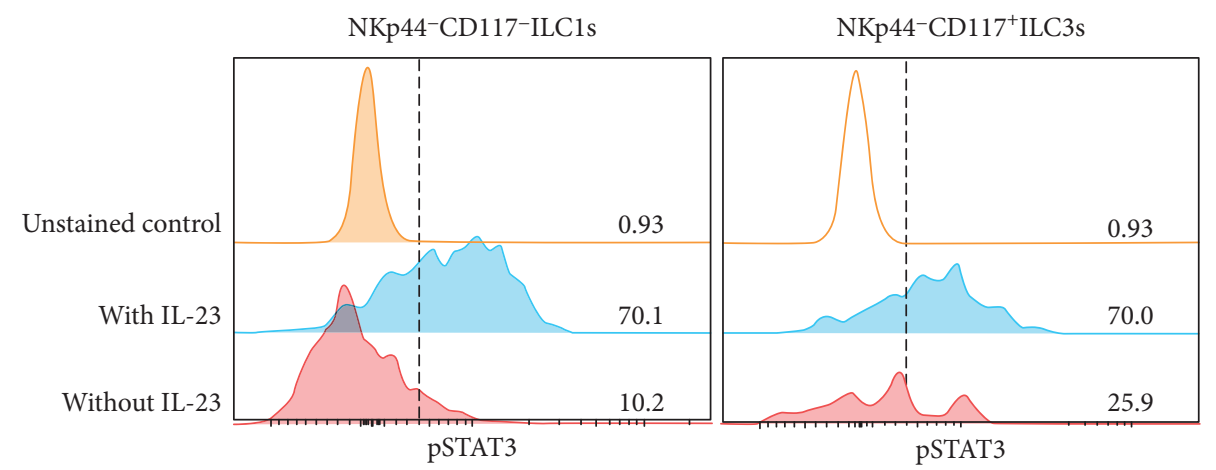

(b)

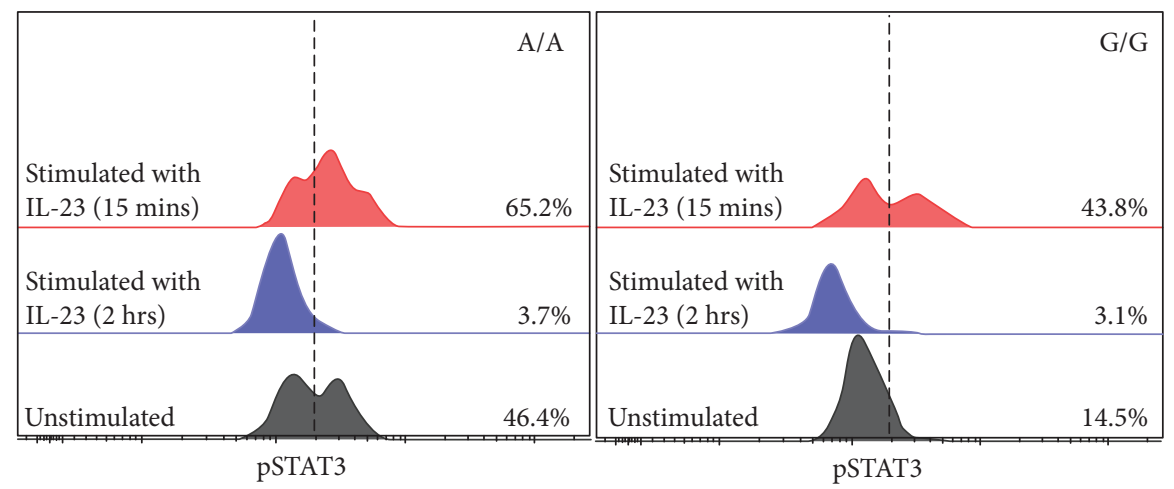

(A)

(B)

(c)

FIgURE 4: Activation of STAT3 signaling in innate lymphoid cells in response to IL-23 stimulation. (a) PBMCs from healthy donor were stimulated by $20 \mathrm{ng} / \mathrm{ml}$ of IL-23 for 15 minutes. After gating Lin ${ }^{-} \mathrm{CD} 45^{+} \mathrm{CD} 127^{+} \mathrm{CRTH} 2^{-}$cells, the ILC population was further gated based on CD117 and NKp44 expression. The CD117 ${ }^{+} \mathrm{NKp} 44^{-}$ILC3 population (NCR ILC3 phenotype) was induced by IL-23 stimulation. (b) IL-23 activated STAT3 signaling in both the CD117 NKp44 ILC1 and CD $117^{-} \mathrm{NKp} 44^{-}$ILC3 subsets. (c) After gating $\mathrm{Lin}^{-} \mathrm{CD} 45^{+} \mathrm{CD} 56^{+} \mathrm{CD} 127^{+} \mathrm{CRTH} 2^{-}$ILCs, the tyrosine phosphorylation level of STAT3 was evaluated in ILCs. Donor with A/A genotype was shown in (A), and donor with G/G genotype was demonstrated in (B). The pSTAT3 level after 15 mins or 2 hrs stimulation of IL-23 indicated there was no delayed dephosphorylation in donors, suggesting risky allele " $A$ " carriage does not affect the dephosphorylation of STAT3 in ILCs. 
remission, relapse rates at one, two, five, and ten years are estimated at $20 \%, 40 \%, 67 \%$, and $76 \%$, respectively [30]. Therefore, a better understanding of $\mathrm{CD}$ pathogenesis is needed in order to select more specific and effective treatment regimens. There are several newly approved small molecule inhibitors targeting JAK-STAT signaling; however, the responsiveness varies by individual [31]. This study was mainly focused on investigating the role of STAT3 in the pathogenesis and progression of Crohn's disease. We positively correlated the prevalence of the STAT3 rs744166 risky allele "A" with disease severity in terms of surgery numbers (Figure 1). In addition, we observed an increased level of stat3 mRNA, STAT3-related gene expression, as well as activation of STAT3 signaling (phosphorylated STAT3) in inflamed ileum tissues of CD patients compared to unaffected ileum (Figures 2 and 3). To investigate STAT3 activity in the innate lymphoid cells of particular interest, we then specifically examined pSTAT3 in each of the ILC subsets in response to IL-23 stimulation (Figure 4). Notably, we found that STAT3 signaling was not only activated in the NCR ILC3 subset but also activated in $\mathrm{Lin}^{-} \mathrm{CD} 45^{+} \mathrm{CRTH} 2^{-} \mathrm{CD} 127^{+} \mathrm{CD} 117^{-} \mathrm{NKp} 44^{-}$cells. Moreover, we illustrated the carriage of STAT3 genotypic variant results in an increased basal and IL-23-stimulated STAT3 tyrosine phosphorylation, but it does not affect the dephosphorylation of STAT3 in ILCs.

Previous studies have focused largely on the role of adaptive immunity and how it drives the pathogenesis as well as progression of Crohn's disease. The research on innate immune responses have been continuously expanded over the past two decades [32]. As the important tissue-resident guardian, innate lymphoid cell has been investigated in both steady state and diseased state, suggesting the important role of ILCs in mucosal immunity [33]. Specifically in Crohn's disease patients, our previously published study showed that the accumulation of IFN- $\gamma$-producing $\mathrm{Lin}^{-} \mathrm{CD} 45^{+-}$ $\mathrm{CRTH} 2^{-} \mathrm{CD} 127^{+} \mathrm{CD} 117^{-} \mathrm{NKp} 44^{-}$cells in the inflamed terminal ileum of CD patients correlates with disease severity [13]. However, the paradox we have observed is an increased STAT3 activity along with decreased ILC3 numbers in the inflamed tissues of CD patients. Bernink et al. not only proposed that IL-23 triggered ILC1-to-ILC3 transdifferentiation but also pointed out that sustained exposure of IL-23 was able to promote the reverse conversion [15]. Other groups also pointed out that IL-23 responsive ILC3s induced pathogenesis of neonatal intestinal inflammation [34]. Additionally, it has been shown that STAT3 phosphorylation in ILC3s was suppressed by the presence of $\mathrm{T}_{\text {reg }}$ and Th17 cells [35]. In this study, we revealed that the STAT3 genotypic variant $r s 744166$ risk allele "A" was associated with increased basal and IL-23 triggered the pSTAT3 level in ILCs, but there was no impact on the dephosphorylation of STAT3 in those genetically susceptible individuals. Taken together, we suggested that investigation of STAT3 signaling in a cell-specific manner is needed for future researches. IL-23-induced activation of STAT3 signaling in CD117 NKp44 ILC subset suggested that STAT3 plays a role in the initiation of ILC plasticity and also potentially involves in regulating $\mathrm{Lin}^{-} \mathrm{CD} 45^{+} \mathrm{CRTH} 2^{-} \mathrm{CD} 127^{+-}$
CD117 NKp44- ILC1 function. More in depth investigation on exact gene regulation of STAT3 in ILCs is necessary to further explore the exact molecular mechanism of ILC plasticity and heterogeneity property.

\section{Conclusion}

Overall, this study illustrated that increased STAT3 activity was associated with the pathogenesis and progression of Crohn's disease. Particularly, activated STAT3 signaling in IL-23 responsive ILCs may lead to the chronic relapse of CD. Notably, individuals carrying STAT3 rs744166 "A” risk allele exhibited increased basal and IL-23-stimulated STAT3 tyrosine phosphorylation in the peripheral ILCs. The results provide a novel concept of a potential mechanism of ILC plasticity and also contribute to potential mechanistic strategies for use in personalized medicine in the treatment of Crohn's disease patients.

\section{Data Availability}

The genotyping, clinical outcomes of patients, and flow cytometry data used to support the findings of this study are available from the corresponding author upon request.

\section{Conflicts of Interest}

The authors have no conflicts of interest regarding the publication of this paper.

\section{Acknowledgments}

This study was approved by the University of Florida Institutional Review Board. We would like to thank Dr. Iqbal and Dr. Tan for consenting patients and CTSI Biorepository for providing human surgical intestinal tissues. We also would like to thank Dr. Andria Doty, the director of ICBR Cytometry Core at the UF, for flow cytometry training and troubleshooting. Moreover, we appreciated the help from the Molecular Pathology Core at the UF for processing FFPE tissue blocks and IHC staining. Furthermore, we would like to thank Dr. Xinyue Liu for statistical analysis consultation. This work was supported by the Gatorade Trust through funds distributed by the University of Florida, Department of Medicine. This work was also supported by the American Gastroenterological Association (AGA) Research Foundation's Pfizer Pilot Research Award in Inflammatory Bowel Disease.

\section{Supplementary Materials}

Supplementary 1. Supplementary Table 1: clinical characteristics of enrolled Crohn's disease patients.

Supplementary 2. Supplementary Table 2: list of human immune response genes.

Supplementary 3. Supplementary Table 3: quantitative expression levels of human immune response genes.

Supplementary 4. Supplementary Figure 1: RT-PCR of stat3 mRNA level in PBMCs from donor and Crohn's disease patients. 
Supplementary 5. Supplementary Figure 2: positive and negative controls of pSTAT3-Y705 IHC staining.

Supplementary 6. Supplementary Figure 3: gating strategy of ILC subsets using healthy donor PBMCs.

\section{References}

[1] M. F. Neurath, "Current and emerging therapeutic targets for IBD," Nature Reviews Gastroenterology \& Hepatology, vol. 14, no. 5, pp. 269-278, 2017.

[2] Y. Z. Zhang and Y. Y. Li, "Inflammatory bowel disease: pathogenesis," World Journal of Gastroenterology, vol. 20, no. 1, pp. 91-99, 2014.

[3] R. Boyapati, J. Satsangi, and G. T. Ho, "Pathogenesis of Crohn's disease," F1000Prime Reports, vol. 7, 2015.

[4] R. B. Sartor, "Mechanisms of disease: pathogenesis of Crohn's disease and ulcerative colitis," Nature Clinical Practice Gastroenterology \& Hepatology, vol. 3, no. 7, pp. 390-407, 2006.

[5] Y. Huang and Z. Chen, "Inflammatory bowel disease related innate immunity and adaptive immunity," American Journal of Translational Research, vol. 8, no. 6, pp. 2490-2497, 2016.

[6] A. Geremia, P. Biancheri, P. Allan, G. R. Corazza, and A. Di Sabatino, "Innate and adaptive immunity in inflammatory bowel disease," Autoimmunity Reviews, vol. 13, no. 1, pp. 310,2014

[7] C. Dai, M. Jiang, and M.-J. Sun, "Innate immunity and adaptive immunity in Crohn's disease," Annals of Translational Medicine, vol. 3, no. 3, pp. 34-34, 2015.

[8] C. S. N. Klose and D. Artis, "Innate lymphoid cells as regulators of immunity, inflammation and tissue homeostasis," Nature Immunology, vol. 17, no. 7, pp. 765-774, 2016.

[9] E. Vivier, D. Artis, M. Colonna et al., "Innate lymphoid cells: 10 years on," Cell, vol. 174, no. 5, pp. 1054-1066, 2018.

[10] G. F. Sonnenberg and D. Artis, "Innate lymphoid cells in the initiation, regulation and resolution of inflammation," Nature Medicine, vol. 21, no. 7, pp. 698-708, 2015.

[11] A. Diefenbach, "Innate lymphoid cells in the defense against infections," European Journal of Microbiology \& Immunology, vol. 3, no. 3, pp. 143-151, 2013.

[12] M. Colonna, "Innate lymphoid cells: diversity, plasticity, and unique functions in immunity," Immunity, vol. 48, no. 6, pp. 1104-1117, 2018.

[13] J. Li, A. L. Doty, A. Iqbal, and S. C. Glover, "The differential frequency of Lineage(-)CRTH2(-)CD45(+)NKp44(-)CD117(CD127(+)ILC subset in the inflamed terminal ileum of patients with Crohn's disease," Cellular Immunology, vol. 304-305, pp. 63-68, 2016.

[14] M. Forkel and J. Mjosberg, "Dysregulation of group 3 innate lymphoid cells in the pathogenesis of inflammatory bowel disease," Current Allergy and Asthma Reports, vol. 16, no. 10, p. 73, 2016.

[15] J. H. Bernink, L. Krabbendam, K. Germar et al., "Interleukin12 and -23 control plasticity of CD127(+) group 1 and group 3 innate lymphoid cells in the intestinal lamina propria," Immunity, vol. 43, no. 1, pp. 146-160, 2015.

[16] A. Geremia and C. V. Arancibia-Cárcamo, "Innate lymphoid cells in intestinal inflammation," Frontiers in Immunology, vol. 8, pp. 1296-1296, 2017.

[17] UK IBD Genetics Consortium, J. C. Lee, D. Biasci et al., "Genome-wide association study identifies distinct genetic contributions to prognosis and susceptibility in Crohn's disease," Nature Genetics, vol. 49, no. 2, pp. 262-268, 2017.

[18] J. Zhang, J. Wu, X. Peng, J. Song, J. Wang, and W. Dong, "Associations between STAT3 rs744166 polymorphisms and susceptibility to ulcerative colitis and Crohn's disease: a meta-analysis," PLoS One, vol. 9, no. 10, article e109625, 2014.

[19] T. A. Willson, B. R. Kuhn, I. Jurickova et al., "STAT3 genotypic variation and cellular STAT3 activation and colon leukocyte recruitment in pediatric Crohn disease," Journal of Pediatric Gastroenterology and Nutrition, vol. 55, no. 1, pp. 32-43, 2012.

[20] K. Sugimoto, "Role of STAT3 in inflammatory bowel disease," World Journal of Gastroenterology, vol. 14, no. 33, pp. 51105114, 2008.

[21] M. Ciofani, A. Madar, C. Galan et al., "A validated regulatory network for Th17 cell specification," Cell, vol. 151, no. 2, pp. 289-303, 2012.

[22] X. Guo, J. Qiu, T. Tu et al., "Induction of innate lymphoid cellderived interleukin-22 by the transcription factor STAT3 mediates protection against intestinal infection," Immunity, vol. 40, no. 1, pp. 25-39, 2014.

[23] L. C. Rankin, M. J. H. Girard-Madoux, C. Seillet et al., "Complementarity and redundancy of IL-22-producing innate lymphoid cells," Nature Immunology, vol. 17, no. 2, pp. 179-186, 2016.

[24] O.. I. Koues, P.. L. Collins, M. Cella et al., "Distinct gene regulatory pathways for human innate versus adaptive lymphoid cells," Cell, vol. 165, no. 5, pp. 1134-1146, 2016.

[25] N. Serafini, C. A. J. Vosshenrich, and J. P. Di Santo, "Transcriptional regulation of innate lymphoid cell fate," Nature Reviews Immunology, vol. 15, no. 7, pp. 415-428, 2015.

[26] L. R. Ferguson, D. Y. Han, A. G. Fraser et al., "Genetic factors in chronic inflammation: single nucleotide polymorphisms in the STAT-JAK pathway, susceptibility to DNA damage and Crohn's disease in a New Zealand population," Mutation Research/Fundamental and Molecular Mechanisms of Mutagenesis, vol. 690, no. 1-2, pp. 108-115, 2010.

[27] G. Can, A. Tezel, H. Gurkan et al., "Investigation of IL23R, JAK2, and STAT3 gene polymorphisms and gene-gene interactions in Crohn's disease and ulcerative colitis in a Turkish population," The Turkish Journal of Gastroenterology, vol. 27, no. 6, pp. 525-536, 2016.

[28] G. Pickert, C. Neufert, M. Leppkes et al., "STAT3 links IL-22 signaling in intestinal epithelial cells to mucosal wound healing," The Journal of Experimental Medicine, vol. 206, no. 7, pp. 1465-1472, 2009.

[29] S. A. van de Pavert and E. Vivier, "Differentiation and function of group 3 innate lymphoid cells, from embryo to adult," International Immunology, vol. 28, no. 1, pp. dxv052-dxv042, 2015.

[30] A. Lapidus, O. Bernell, G. Hellers, and R. Lofberg, "Clinical course of colorectal Crohn's disease: a 35-year follow-up study of 507 patients," Gastroenterology, vol. 114, no. 6, pp. 11511160, 1998.

[31] L. C. S. De Vries, M. E. Wildenberg, W. J. De Jonge, and G. R. D'Haens, "The future of Janus kinase inhibitors in inflammatory bowel disease," Journal of Crohn's and Colitis, vol. 11, no. 7, pp. 885-893, 2017.

[32] N. Li and R.-H. Shi, "Updated review on immune factors in pathogenesis of Crohn's disease," World Journal of Gastroenterology, vol. 24, no. 1, pp. 15-22, 2018. 
[33] A. Ignacio, C. N. S. Breda, and N. O. S. Camara, "Innate lymphoid cells in tissue homeostasis and diseases," World Journal of Hepatology, vol. 9, no. 23, pp. 979-989, 2017.

[34] L. Chen, Z. He, E. Slinger et al., "IL-23 activates innate lymphoid cells to promote neonatal intestinal pathology," Mucosal Immunology, vol. 8, no. 2, pp. 390-402, 2015.

[35] K. Mao, A. P. Baptista, S. Tamoutounour et al., "Innate and adaptive lymphocytes sequentially shape the gut microbiota and lipid metabolism," Nature, vol. 554, no. 7691, pp. 255259, 2018. 


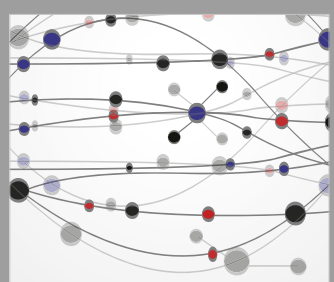

The Scientific World Journal
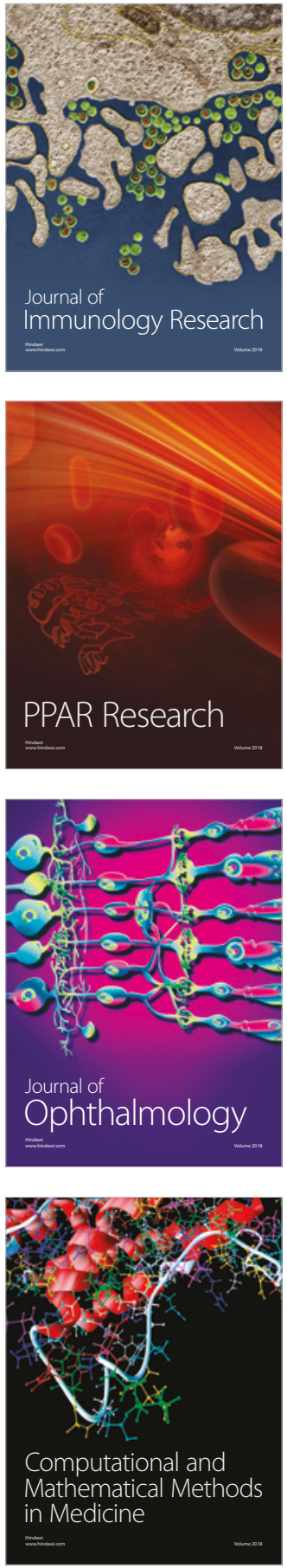

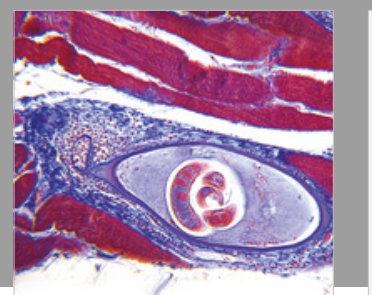

Gastroenterology Research and Practice

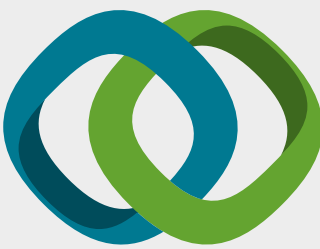

\section{Hindawi}

Submit your manuscripts at

www.hindawi.com
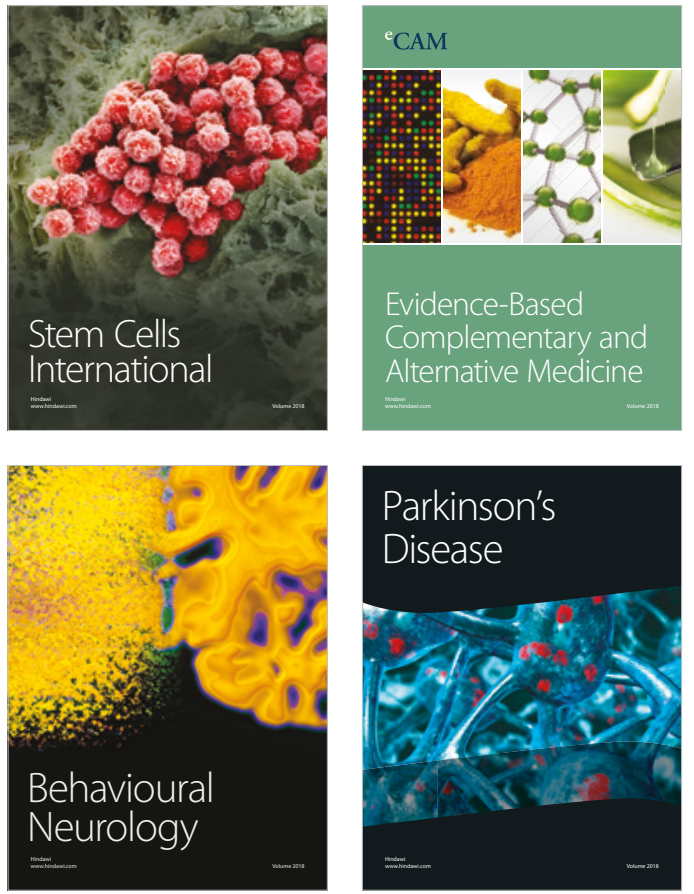

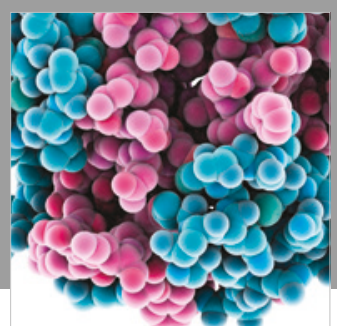

ournal of

Diabetes Research

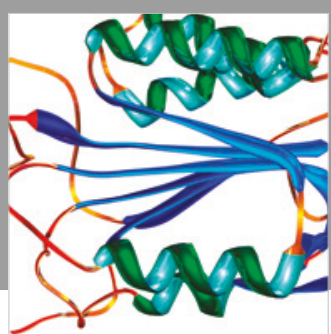

Disease Markers
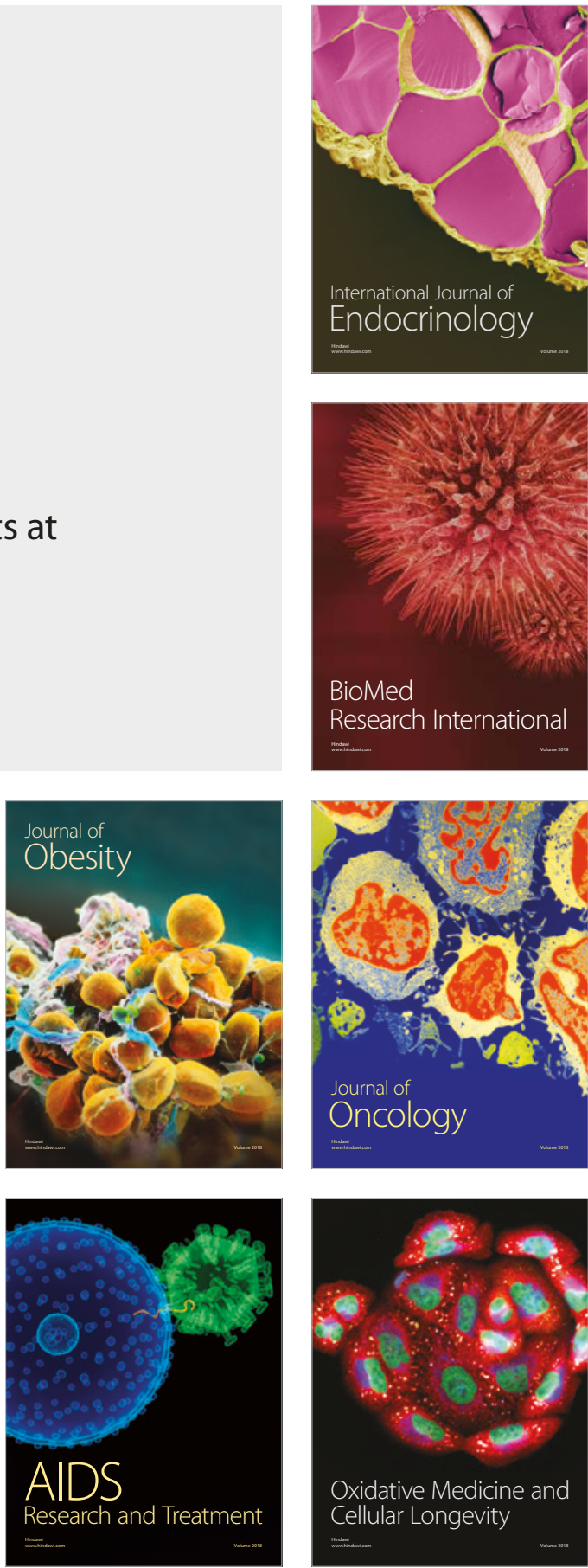